\title{
Lead Projectile Fragmentation on Aluminum Target: Improved Experimental Results
}

\author{
Gul Sher, Mukhtar Ahmed Rana, Muhammad Ikram Shahzad \\ Physics Division, Pakistan Institute of Nuclear Science and Technology, P.O. Nilore, Islamabad, Pakistan \\ E-mail: gsher12000@yahoo.com
}

Received March 29, 2011; revised April 7, 2011; accepted April 11, 2011

\begin{abstract}
We investigate $158 \mathrm{~A} \mathrm{GeV}{ }^{207} \mathrm{~Pb}$ projectile fragmentation on $\mathrm{Al}$ target using $\mathrm{CR}-39$ nuclear track detectors. A stack containing target-detectors assembly was irradiated at SPS-CERN. After chemical etching, detectors were scanned using an optical microscope to collect the data in the form of etched cone heights. From the cone height measurements, total and partial charge changing cross sections of ${ }^{207} \mathrm{~Pb}$ projectiles on aluminum target are determined. The results are compared with both relevant published measurements available in the literature and model predictions. Odd-even effect in the formation of the fragments of $158 \mathrm{~A} \mathrm{GeV}{ }^{207} \mathrm{~Pb}$ projectiles is observed in a consistent and a clear manner over the observed range of fragments $(Z=63-81)$. This is achieved by using optimized etching and track measurement conditions.
\end{abstract}

Keywords: Relativistic Energy, Projectile Fragmentation, CR-39 Detectors

\section{Introduction}

Nuclear projectile fragmentation at relativistic energies provides an insight into some aspects of nuclear structure, especially the peripheral structure of the nucleus. In central and peripheral nuclear collisions, the fragmentation process leads to the emission of fragments with a wide range of masses. Experimental results on fragmentation properties of relativistic energy projectiles are relevant for nuclear physics, cosmic ray physics and astrophysics. Fragmentation cross sections are required to understand and evaluate composition of the cosmic rays propagation through the interstellar medium [1,2]. At relativistic energy, projectile fragmentation on different targets has been studied by a number of authors using both active and passive detection techniques [3-8].

CR-39 $\left(\mathrm{C}_{12} \mathrm{H}_{18} \mathrm{O}_{7}\right)$ nuclear track detectors are manufactured by Intercast Europe Co. of Parma Italy. These detectors have been used for the study of different projectiles and targets interaction at relativistic energy [9-13]. Previously, we have presented $158 \mathrm{~A} \mathrm{GeV}$ fragmentation $[10,11]$ studies on $\mathrm{Bi}$ and $\mathrm{Cu}$ targets using the most sensitive CR-39 track detector. In this paper, we present $\mathrm{Pb}$ projectile fragmentation on $\mathrm{Al}$ target using the welloptimized experiments with the experience of previous experiments. Optimized experimental etching conditions provided the improved results compared with our previous investigations in a way that odd-even effect has been observed in a clear and consistent manner over the observed range of fragments of $158 \mathrm{~A} \mathrm{GeV} \mathrm{Pb}$ nuclei $(\mathrm{Z}=$ $63-81)$ in comparison with our previous reports $[10,11]$. This is an important observation which is achieved through optimization of experimental conditions details of which are given in sections 2 and 3. Section 2 is about experimental detail and explains the methodology employed for detector calibration and calculation charge changing cross sections. Results are discussed in section 3 whereas the last section contains conclusions.

\section{Experiments}

A stack containing Al target sandwiched with CR-39 detector sheets of the size $(10 \mathrm{~cm} \times 10 \mathrm{~cm} \times 1 \mathrm{~mm})$ was exposed to $158 \mathrm{~A} \mathrm{GeV} \mathrm{Pb}$ ions at SPS beam of CERN. The exposure was performed at normal incidence with a fluence of about 1500 ions $/ \mathrm{cm}^{2}$. The total number of lead ions in the stack was about $7.8 \times 10^{4}$ distributed in eight spills. In the exposure stack four CR-39 sheets were placed upstream the target and almost 39 sheets downstream the target. In order to avoid multifragmentation, appropriate gaps were provided in the detectors placed downstream the target in the stack, size of the gaps was nearly $2 \mathrm{~cm}$ between the detector foils placed close to the target and gaps were increased as the stack length. 
In order to scan, we selected one CR-39 sheet upstream the target and other two downstream the target (one close to the target and other from last pairs in the stack). These detectors were etched in $4 \mathrm{~N} \mathrm{KOH}$ aqueous solution at $45^{\circ} \mathrm{C}$ for $72 \mathrm{~h}$.

In order to improve and implement appropriate etching conditions a considerable emphasis was given to the important factors contributing in the etching process, the concentration of active etching molecules in the etching solution an effective and continuous stirring was maintained using a calibrated motorized stirring system during the etching process to produce a sort of convection in the etching bath to avoid buildup etch products produced from detector surface in developing tracks. The variation in the temperature was not more than $\pm 1^{\circ} \mathrm{C}$ to maintain control and stability. Concentration of etchant was kept effectively constant under specific etching conditions by minimizing the water evaporation from the etching solution during the etching.

After etching process the beam ions and the fragments registered in the detector placed after the target appeared as etched cones on both sides of the detectors. The detectors were scanned using optical microscope at a magnification of $400 \times$. Using the built-in cone height measuring unit of optical microscope the cone lengths of track events have been measured with an accuracy of $\pm 1 \mathrm{~m}$. Well optimized experimental procedures and precautions, especially for the chemical etching, were adopted throughout the experiments and track measurements. Bases of such procedures and precautions are described elsewhere in detail [11].

\section{Results and Discussion}

\subsection{Charge Identification and Detector Calibration}

The etched cones of vertically incident beam particles and the fragments are of circular shape and completely black in direct illumination. The background objects are shallow and appear as black circular objects with a brighter centre are not considered. Target fragments can be resolved based on their short range. The central brightness can be used to separate particle tracks from background. Figure 1 shows the distribution of cone height for beam ions and the fragments registered in a detector placed after the target. The ionizing particles traversing through an insulating material create a narrow path of intense damage on an atomic scale [12].

The damaged region in the detector can be revealed and enlarged by treating with a proper chemical reagent to become visible under an optical microscope. During the etching process, the track etch rate $v_{\mathrm{T}}$ exceeds the bulk etch rate $v_{\mathrm{B}}$ producing a conical etch-pit at the point

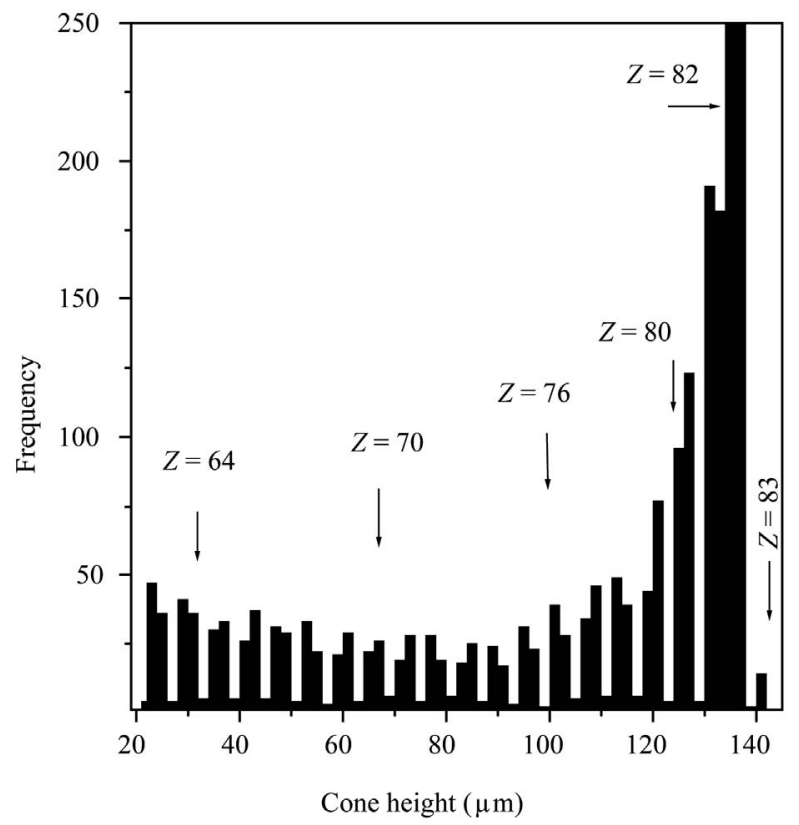

Figure 1. Average cone heights distribution of $\mathrm{Pb}$ ions and the fragments produced in interaction with the target observed using CR-39 detector placed after the Al target, arrows indicate charge (atomic number) assigned to peak.

of entrance and exit of the particle. The size and shape of such cones depend on the energy loss of the incident particle; the measurement of the cone base area or heights allows, through an appropriate calibration, to determine the characteristics of the particle.

The focused surface of the opening of the etch-pits looks like a dark, circular of well defined diameter. The CR-39 detectors, placed upstream the targets registered the total number of incident $\mathrm{Pb}$ ions and detectors placed after the target recorded both the survived $\mathrm{Pb}$ ions and the fragments produced in the interaction of $\mathrm{Pb}$ ions with the target. Since the beam exposure is normally incident to the detector surface, the beam ions and the fragments produced as a result of fragmentation almost maintain their longitudinal velocity along the direction of beam ions and a sequence of etch cones is produced on the subsequent detector surfaces.

At relativistic energies the base area of the post etching cones depends only on charge $\mathrm{Z}$, therefore peaks of measured etched cone heights correspond to different charges. This method is applicable as long as the projectile peak and the fragment peaks are clearly separated. It is reported in [13] that the etched cone diameter is sensitive to low $\mathrm{Z}$ ions, and cone height to high $\mathrm{Z}$ ions. The average values of the cone heights distribution observed in both the detectors is displayed in the form of well separated peaks, which are assigned to a charge value $63 \leq$ $\mathrm{Z} \leq 82$ by counting downward beginning with the beam peak $Z=82$ (we assume the charge assigned to the frag- 
ment as the atomic number of the fragment. In reality, charge of the fragment can be lower than the atomic number $\mathrm{Z}$ in a particular case when fragments have lowered their energy and collected electrons from the target which reduces their charge from the maximum possible charge. For the simplicity the atomic number of fragments is shown in Figure 1.

The charge resolution is related to cone height resolution. In view of different units of these quantities relative charge resolution is equated to relative length resolution using the relation

$$
\sigma_{Z}=\left(\sigma_{L}\right) /\left(\delta_{L} / \delta_{Z}\right)
$$

where $\sigma_{L}$ is the standard deviation in the length of individual peaks assigned to charge value. The plot of charge $Z$ verses cone lengths $L$ is shown in Figure 2. The ratio $\delta_{L} / \delta_{Z}$ is the slope of the plot.

The energy deposited by the charged particle to form the latent track is the Restricted Energy Loss (REL).The response of the detectors to relativistic ions is characterized by the correlation of parameter $p\left(v_{T} / v_{B}\right)$, and $R E L$ from the calibration curve. Since track etch rate $v_{T}$ is a function of REL, which depends on the charge and energy of the incident projectile ion, for relativistic energy ions the $R E L$ remains approximately constant therefore, it is considered that the diameter and length of etched cone are function of ion charge only as reported by [14].

The cylindrical cone length $L$ is related to etch rate ratio ' $p$ ' through the equation

$$
L=v_{B} t(p-1)
$$

where' $t$ ' is etching time. The track etch rate $\left(v_{T}\right)$ of the

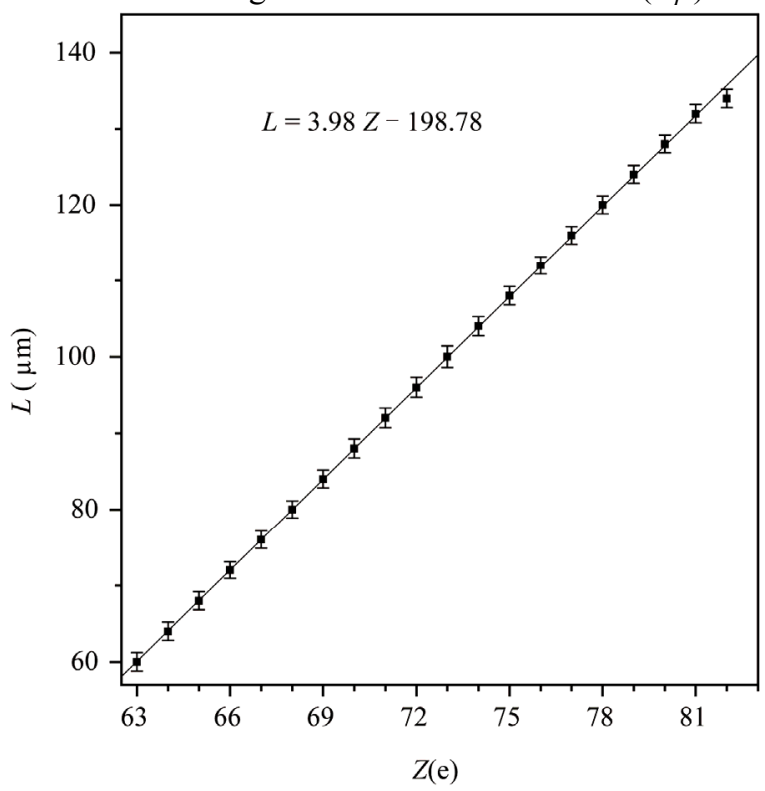

Figure 2. Correlation plot of the charges to the mean etched cone lengths measured in detector placed after the target. cones produced in the detector can be calculated from the relation,

$$
v_{T}=v_{B}+L / t
$$

where $L$ is the cone height, $t$ is the etching time and $v_{B}$ is the bulk etch rate of the detector measured by the change in the thickness of the detector before and after etching of the detector. The refractive index of the CR-39 was experimentally determined and was used for the determination of the total height of cones.

For the resolution of higher charges the cone heights are associated with different charges. The charge values of fragments with almost identical $\beta$ are associated with specific $R E L$ values while the lengths are converted to ' $p$ ' values using Equation (3).

The relation between $R E L$ and ' $p$ ' is specific to the etching condition, because the value of ' $p$ ' is experimentally measured for the given etching conditions when unknown ion interacts on the detector, it is, therefore, possible to assign it an $R E L$ value. The REL vs. $p$ graph as shown in Figure 3 constitute a calibration curve. The function $R E L$ defined as

$$
R E L=(\mathrm{d} E / \mathrm{d} x)_{E<E_{\text {max. }}=200 \mathrm{eV}}
$$

For particles with $\beta_{C}>10^{-2} \mathrm{c}$, the $R E L$ is a fraction of the electronic energy loss, leading to $\delta$ rays with energies lower than $E_{\max }$, with $E_{\max }=200 \mathrm{eV}$ for CR-39 [15]. If the $R E L$ of a particle passing through a stack of detectors

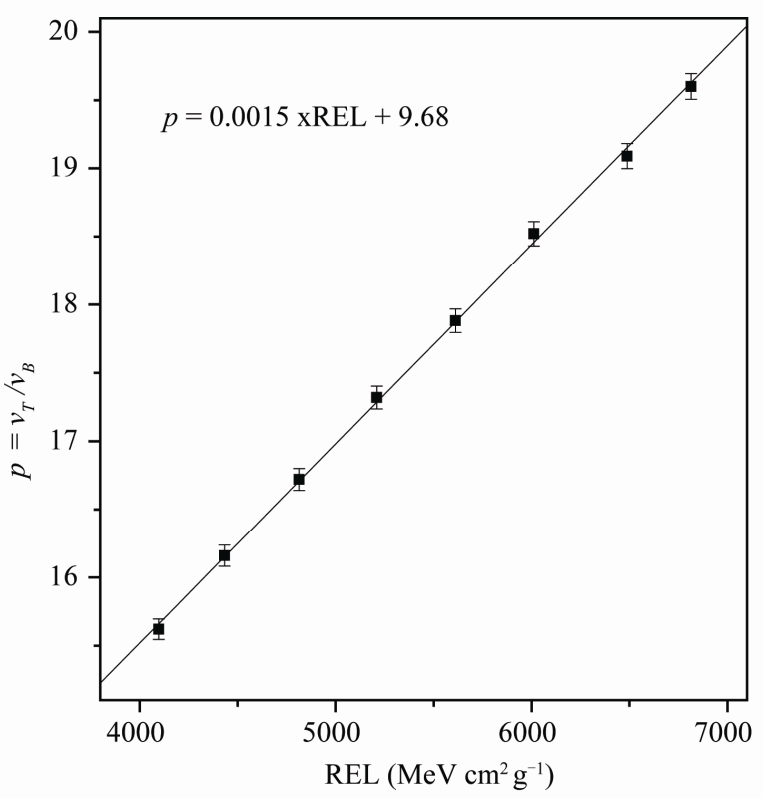

Figure 3. The calibration curve of reduced etch rate $p$ vs $R E L$ value. The uncertainties in bulk etch rate measurement yield error in the reduced etch rate $p$. The points are the experimental data and the line is the fit to the data point. The fitted equation is valid in the $R E L$ range $\sim 4200-7000$ $\mathrm{MeV} \mathrm{cm}^{2} / \mathrm{g}$ under present etching conditions. 
is constant along its trajectory and the same etching conditions are applied to all the detectors in the stack, the track etching rate $v_{T}$ is constant and identical cones are formed on all crossed detector surfaces.

Figure 4 shows the track length and charge resolutions for the fragments of $158 \mathrm{~A} \mathrm{GeV} \mathrm{Pb}$ projectiles produced in collisions of the projectile beam with Al target. The dotted horizontal line shows the mean values of track length and charge resolutions. We have also analyzed the distributions of track length (Figure 5(a) and charge resolutions (Figure 5(b)). Values of $\chi^{2} /$ dof and squared regression coefficients $\mathrm{R}^{2}$ for the Gaussian fits on both distributions are given in the respective plots in Figure 4. Peak values of the Gaussian fits on track length and charge resolutions in Figure 4 agree well with the respective arithmatic mean values in Figure 4.

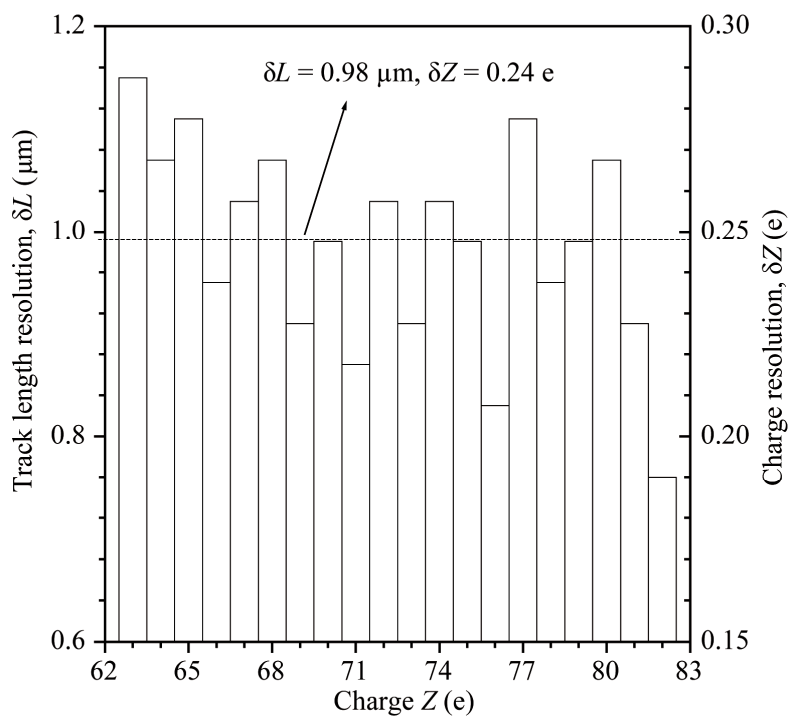

Figure 4. Track length and charge resolutions for the measured fragments of 158A $\mathrm{GeV} \mathrm{Pb}$ projectiles.

\subsection{Charge Changing Cross Section Results}

The probability of projectile charge change in an interaction with the target nucleus can be estimated by measuring the survival fraction of beam ions during the projectile fragmentation on target. The number of incident and survived beam ions is determined considering the data of etched cone before and after the target. The total charge changing cross section contains both the nuclear and electromagnetic cross section contribution expressed as

$$
\sigma_{\text {tot }}^{\exp }=\sigma_{\text {nuc }}^{\text {exp }}+\sigma_{\text {em }}^{\exp }
$$

The total charge changing cross section is determined using the following expression

$$
\sigma_{\text {tot }}^{\text {exp }}=\frac{A_{T}}{N_{A} \rho_{T} t_{T}} \ln \left[\frac{N_{i}}{N_{s}}\right]
$$
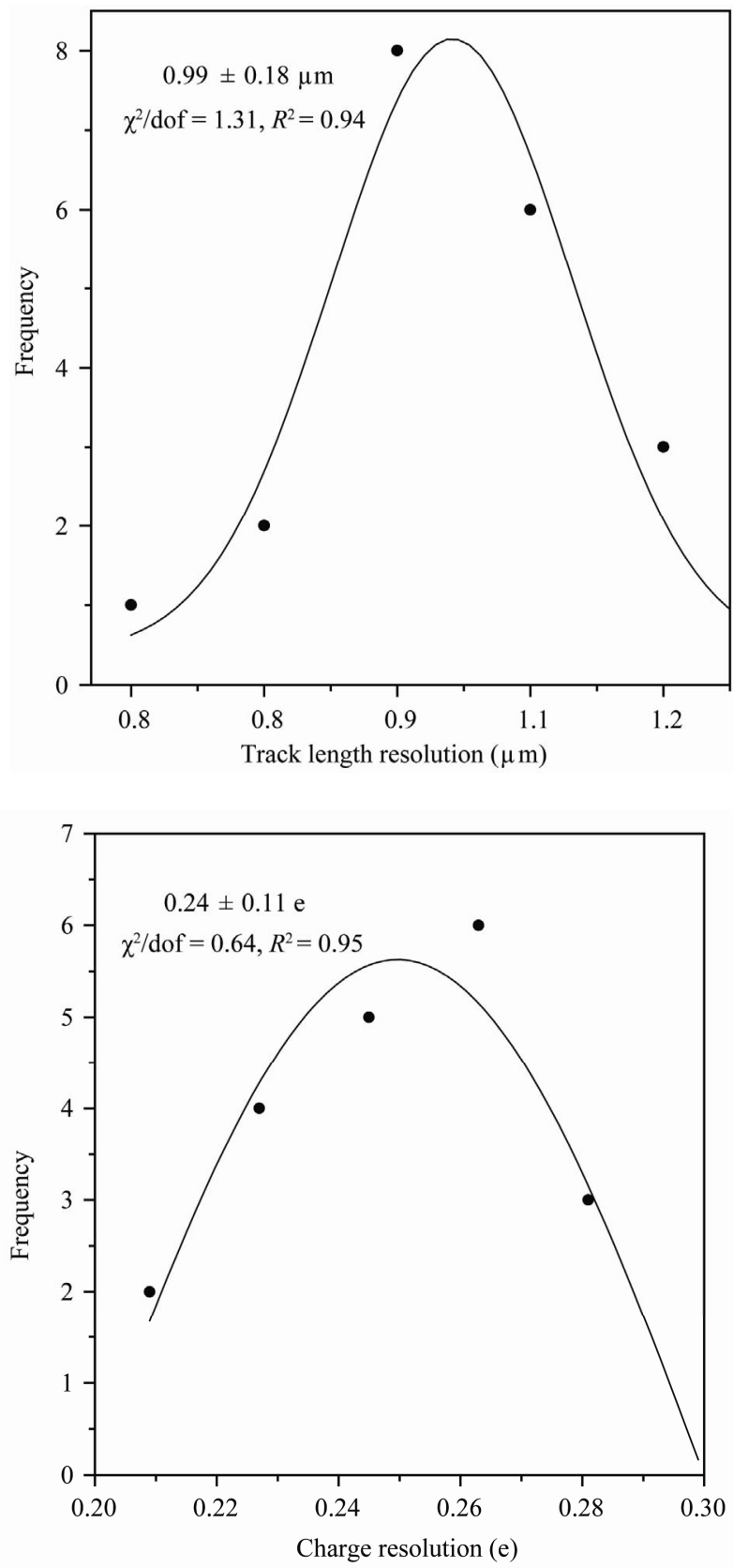

Figure 5. The comparison of distributions of measured track length resolution $\delta L$ and assigned charge resolution $\delta Z$ for the fragments of $63 \leq Z \leq 82$.

where $A_{T}$ is the nuclear mass of the target. $N_{i}$ and $N_{s}$ are the number of incident beam ions before and after the target respectively, $\rho_{T}\left(\mathrm{~g} / \mathrm{cm}^{3}\right)$ is the target density; $t_{T}(\mathrm{~cm})$ is the thickness of the target and $N_{A}$ is Avogadro's number. In the simplest model, the reaction cross sections are assumed to be proportional to the geometrical area of the interacting nuclei $\left(\pi R^{2}\right.$, where $R$ is the sum of interacting nuclei radii). The semi empirical model approximates the total reaction cross section 


$$
\sigma_{\text {tot }}^{\text {th }}=\pi r_{o}^{2}\left(A_{p}^{1 / 3}+A_{T}^{1 / 3}-b\right)^{2}
$$

with parameters $r_{0}=1.35 \mathrm{fm}$, and $b=0.83[16,17]$. The parameter $r_{0}$ is an energy independent effective nuclear radius, $b$ is either an energy independent or energy dependent overlap or transparency parameter, $A_{P}$ and $A_{T}$ are the projectile and target mass numbers respectively.

The experimentally observed total fragmentation cross sections of $\mathrm{Pb}$ projectiles on $\mathrm{Al}$ target, given in Table 1, are found to be comparable, within experimental errors, to the corresponding calculations using the model in Equation (6). Our measurement of cross section is also in good agreement with the similar published results $[5,6]$, whereas data of [18] is somewhat small, which are also given in the Table 1. However, our measured cross section for $\mathrm{Al}$ target is small in comparison with the reported measured results for heavy targets at similar energies $[5,6]$. This is due to the enhanced electromagnetic dissociation (EMD) contribution to the total cross sections for heavy targets nuclei which offer stronger coulomb potential to the penetrating projectiles. The EMD contribution is comparatively small in the present case of Al target. The comparative roles of strong and electromagnetic interactions in the heavy projectile and target collisions at relativistic energies have been discussed in detail in the literature $[2,8]$.

Partial cross sections are measured using the ratio of the number of fragments of a particular observed assigned charge to the number of survived projectiles. The following relationship was employed to determine partial cross sections from the observed data,

$$
\sigma_{\Delta Z}=\frac{A_{T}}{N_{A} \rho_{T} t_{T}}\left[\frac{N_{Z}}{N_{S}}\right]
$$

where $Z=63 e-81 e, N_{Z}$ is the number of fragment nuclei with the charge $Z$ produced in the target, $N_{S}$ is the number of survived projectiles and $\rho_{T}, A_{T}, t_{T}, N_{A}$ are the same parameters as mentioned in Equation 5. The partial cross sections $\left(\sigma_{\Delta Z}\right)$ is found to follow the power law in terms of charge change $\Delta Z$,

Table 1. Comparison of measured total fragmentation charge changing cross section with theoretically calculated

\begin{tabular}{|c|c|c|c|c|c|}
\hline Target & $A_{T}$ & $Z_{T}$ & $\rho_{T} \quad\left(\mathbf{g} / \mathbf{c m}^{3}\right)$ & $\sigma_{t o t}^{\exp } \quad(\mathbf{m b})$ & $\sigma_{\text {tot }}^{\text {th }} \quad(\mathbf{m b})$ \\
\hline \multirow[t]{4}{*}{ Al } & 27 & 13 & $2.692 \pm 0.002$ & $3938 \pm 197^{(a)}$ & $3743^{(a)}$ \\
\hline & & & & $3804 \pm 164^{(b)}$ & $3406^{(\mathbf{b})}$ \\
\hline & & & & $3750 \pm 80^{(\mathrm{c})}$ & - \\
\hline & & & & $3601 \pm 23^{(\mathrm{d})}$ & - \\
\hline
\end{tabular}
and results found in literature for the $\mathrm{Al}$ target.

(a). Present work, (b). [5], (c) [6], (d) [18]

$$
\sigma_{\Delta Z}=\alpha|\Delta Z|^{-\beta}
$$

where $\alpha$ and $\beta$ are fit parameters having values (217 $\pm 18) \mathrm{mb}$ and $(0.42 \pm 0.03)$ respectively. We have plotted measured partial cross sections as a function of projectile charge change in Figure 6. It is observed that cross sections of smallest charge change $\Delta Z=-1$ does not follow the power law, which is thought to be influenced by the electromagnetic dissociation in peripheral collisions. The value of smallest charge change is excluded in the fit and the power law provides a reasonable fit to the other values in data sets with a reasonably good values of $\chi^{2} /$ dof $=1.4$. The number of fragments produced is larger for small projectile charge change $\Delta Z$ and decreases with the increase in $\Delta Z$. The odd-even effect is visible in the cross sections results in Figure 6 for $\Delta Z$ values between 5 and 9 where the dependence of cross section on $\Delta Z$ is very weak. This effect is not observed in cross section results for smaller values of $\Delta Z$ due to strong dependence of cross section on $\Delta Z$.

Partial charge changing cross section results in Figure 6 can be divided into two regimes. First regime sweeps the nuclear charge change $(\Delta Z)$ from 1 to 10 whereas the second regime with $\Delta Z$ higher than 10 . Cross sections show strong dependence on $\Delta Z$ regime I compared with the regime II. Regime I is the dynamic regime and

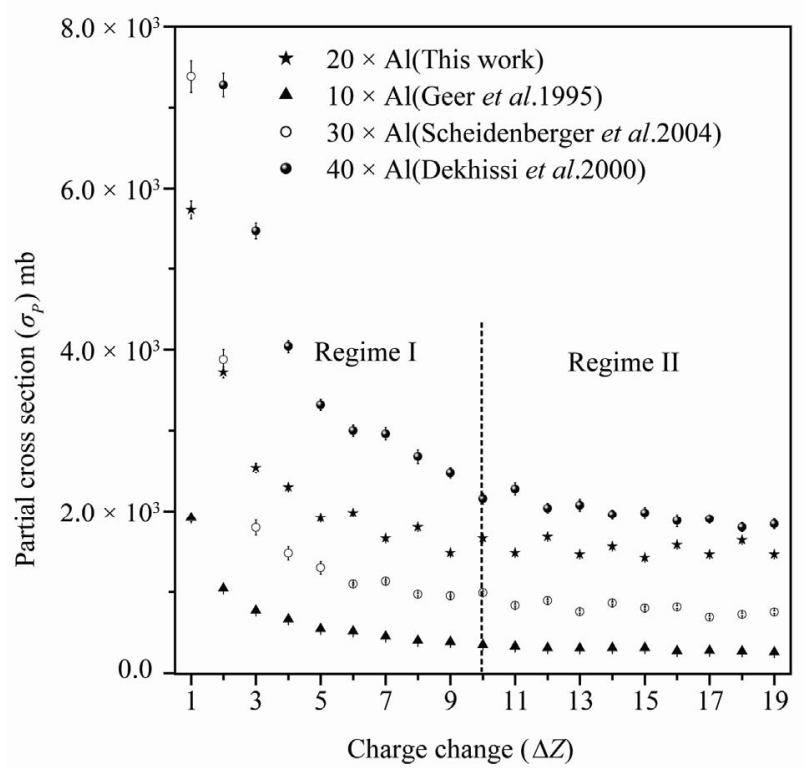

Figure 6. Partial charge-changing cross sections of $158 \mathrm{~A}$ GeV Pb ions $\left(\sigma_{P}\right)$ versus projectile charge change $(\Delta Z)$ in the interaction with $\mathrm{Al}$ target determined using Equation 7. Partial cross section data of 10.6 A GeV Au ions [18] and $158 \mathrm{~A} \mathrm{GeV} \mathrm{Pb}$ ions [5,6] interaction with Al target are shown for comparison with the present measurements. The errors in the measured cross sections include statistical and systematic uncertainties. 
represents the case of peripheral and minor overlap collisions of projectiles with target nuclei. Interaction strength of such collisions varies due to variation in impact parameter. Collisions in the second regime (higher change of $\Delta Z$ ) are central or major overlap collisions which are less probable. Our results (in Figure 6) show that strength of nuclear interaction (of $158 \mathrm{~A} \mathrm{GeV} \mathrm{Pb}$ projectiles with Al target nuclei) achieves a kind of saturation for charge change value higher than 10 .

\section{Conclusions}

Fragmentation process of $158 \mathrm{~A} \mathrm{GeV}{ }^{207} \mathrm{~Pb}$ projectiles on Al target has been studied using CR-39 nuclear track detectors. Measurements of fully resolved etched cone heights corresponding to the projectiles and the fragments produced are used for the determination of the total and partial fragmentation cross sections. The measured total fragmentation cross section is in good agreement with the experimental data of the Al target, but smaller in comparison with the experimental data of heavy targets at similar energy reported in literature [4-6,10]. The results show that the nuclear part of the fragmentation cross section is dominant while the electromagnetic part being very small in the $\mathrm{Pb}$ projectile fragmentation on $\mathrm{Al}$ target. A regular decrease in the measured partial cross sections is observed with the increase in projectile charge change. The partial cross sections follow power law in the charge loss over a wide range of projectile charge change $\Delta Z$.

The measured partial cross sections $\left(158 \mathrm{~A} \mathrm{GeV}{ }^{207} \mathrm{~Pb}\right.$ projectile on $\mathrm{Al}$ target) are larger in comparison with the results of [18] for 10.6 A GeV Au projectiles impinging on the same target. Our measurements are in agreement with the corresponding experimental cross section results of $[5,6]$ for $158 \mathrm{~A} \mathrm{GeV} \mathrm{Pb}$ projectiles penetrating the same target but found smaller than their results of heavy targets. An odd-even effect is observed in a consistent manner, for a section of observed fragments, in the measured partial cross sections of $\mathrm{Pb}$ projectiles on $\mathrm{Al}$ target which shines light on the sensitivity of the of CR-39 detector. Complete analysis of odd-even effect in fragmentation reaction was not possible due to absence of information about neutron number of produced fragments in our experiments. It is worthwhile to mention that detection sensitivity and resolution of CR-39 and other track detectors is strongly influenced by etching conditions. Only the use of well optimized and controlled etch- ing conditions can yield good results.

\section{Acknowledgments}

We express thanks to the technical staff of SPS-CERN for providing beam exposure. Also experimental help of
Mr. Bashir Ahmad Shad Physics Division, PINSTECH is acknowledged. One of authors (MAR) is grateful for the financial support of AS-ICTP (Trieste, Italy) and IAEA for the study visit to AS-ICTP in April 2010.

\section{References}

[1] C. J. Waddington, "The Propagation of Ultraheavy Cosmic-Ray Nuclei Using Energy-Dependent Cross Sections," Astrophysical Journal, Vol. 470, No. 2, 1996, pp. 1218-1226. doi:10.1086/177944

[2] C. A. Bertulani and G. Baur, "Electromagnetic Processes in Relativistic Heavy Ion Collisions," Physical Reports, Vol. 163, No. 5-6, 1988, pp. 299-408.

[3] C. Brechtmann and W. Heinrich, "Fragmentation of ${ }^{16} \mathrm{O}$ at 60, $200 \mathrm{GeV} /$ Nucleon," Zeitschrift fur Physik A, Vol. 330, No. 4, 1988, pp. 407-416.

[4] I. E. Qureshi, M. I. Shahzad, M. T. Javed, S. Manzoor, G. Sher, F. Aleem and H. A. Khan, "Study of Projectile Fragmentation in the Reaction (158A GeV) $\mathrm{Pb}+\mathrm{Pb}$ Using CR-39," Radiation Measurements, Vol. 40, No. 2-6, 2005, pp. 437-441.

[5] H. Dekhissi, G. Giacomelli, G. Mandrioli, S. Manzoor, L. Patrizii, V. Poppa, P. Serra and V. Togo, "Fragmentation Studies of $158 \mathrm{~A} \mathrm{GeV} \mathrm{Pb}$ Ions Using CR-39 Nuclear Track Detectors," Nuclear Physics A, Vol. 662, No. 1-2, 2000, pp. 207-216.

[6] C. Scheidenberger, I. A. Pshenichnov, K. Sümmerer, A. Ventura, et al., "Charge-Changing Interaction of Ultrarelativistic Pb Nuclei," Physical Review C, Vol. 70, No. 1, 2004, pp. 014902 (1-16).

[7] Y. D He and P. B. Price, "Nuclear and Electromagnetic Fragmentation of $2.25 \mathrm{TeV}^{197} \mathrm{Au}$ Nuclei," Zeitschrift Für Physik A Hadrons and Nuclei, Vol. 348, No. 2, 1994, pp. 105-109. doi:10.1007/BF01289597

[8] S. E. Hirzebruch, E. Becker, G. Hüntrup, T. Streibel, E. Winkel and W. Heinrich, "Charge-Changing Interaction of ${ }^{197} \mathrm{Au}$ at $10 \mathrm{GeV} /$ Nucleon in Collision with Targets from H to Pb," Physical Review C, Vol. 51, No. 4, 1995, pp. 2085-2090. doi:10.1103/PhysRevC.51.2085

[9] P. B. Price and Y. D. He, "Behaviour of Nuclear Projectile Fragments Produced in Collision of $14.5 \mathrm{~A} \mathrm{GeV}{ }^{28} \mathrm{Si}$ with $\mathrm{Pb}$ and $\mathrm{Cu}$ Targets," Physical Review C, Vol. 43, No. 2, 1991, pp. 835-845. doi:10.1103/PhysRevC.43.835

[10] G. Sher, M. I. Shahzad and M. Hussain, "Fragmentation of (158A GeV) Pb Ions with Bi Target," Radiation Measurements, Vol. 42, No. 10, 2007, pp. 1692-1695. doi:10.1016/j.radmeas.2007.09.006

[11] M. A. Rana and Shahid Manzoor, "Examining the Fragmentation of 158A GeV Lead Ions on Copper Target: Charge-Changing Cross Sections," Radiation Measurements, Vol. 43, No. 8, 2008, pp. 383-1389.

[12] M. A. Rana, "How to Achieve Precision and Reliability in Experiments Using Nuclear Track Detection Technique?" Nuclear Instruments and Methods in Physics Research Section A, Vol. 592, No. 3, 2008, pp. 354-360. doi:10.1016/j.nima.2008.04.025 
[13] R. L. Fleischer, P. B. Price and R. M. Walker, "Nuclear Tracks in Solids," University of California Press, Berkeley, 1975.

[14] G. Giacomelli, M. Giorgini, G. Mandrioli, S. Manzoor, L. Patrizii, V. Popa, P. Serra, V. Togo and E. C. Vilela, "Extended Calibration of a CR-39 Nuclear Track Detector with $158 \mathrm{~A} \mathrm{GeV}^{207} \mathrm{~Pb}$ Ions," Nuclear Instruments and Methods in Physics Research Section A, Vol. 411, No 1, 1998, pp. 41-45. doi:10.1016/S0168-9002(98)00270-8

[15] J. Dreute, W. Heinrich, G. Rusch and B. Wiegel, "Fragmentation of Gold Projectiles with Energies of 200-980 $\mathrm{MeV} / \mathrm{Nucleon}$. I. Experimental Method Charge Yield and Transverse Momenta," Physical Review C, Vol. 44, No. 3, 1991, pp. 1056-1064.
[16] D. L. Henshaw, N. Griffiths, Otto A. L. Landen and E. V. Benton, "A Method of Producing Thin CR-39 Plastic Nuclear Track Detectors and Their Application in Nuclear Science and Technology," Nuclear Instruments and Methods, Vol. 180, No. 1, 1981, pp. 65-77. doi:10.1016/0029-554X(81)90011-2

[17] H. L. Bradt and B. Peters, "The Heavy Nuclei of the Primary Cosmic Radiation," Physical Review, Vol. 77, No. 1, 1950, pp. 54-70. doi:10.1103/PhysRev.77.54

[18] G. D. Westfall, L. W. Wilson, P. J. Lindstrom, H. J. Crawford, D. E. Greiner and H. H. Heckman, "Fragmentation of Relativistic ${ }^{56} \mathrm{Fe}$," Physical Review C, Vol. 19, No. 4, 1979, pp. 1309-1323. doi:10.1103/PhysRevC.19.1309 